\title{
A prospective longitudinal study of depression, cognitive decline, and physical impairments in patients with Parkinson's disease
}

\author{
Sergio E Starkstein, Helen S Mayberg, Ramón Leiguarda, Thomas J Preziosi, \\ Robert G Robinson
}

\begin{abstract}
A consecutive series of 105 patients with Parkinson's disease were examined for the presence of affective disorders, cognitive deficits, and impairments in activities of daily living (ADLs); 92 received the same evaluation 12 months after the initial examination. On the basis of the initial psychiatric findings, patients were divided into major, minor, and non-depressed groups. Patients with major depression showed a significantly greater cognitive decline, deterioration in ADLs, and further advance through the Hoehn and Yahr stages than patients with either minor depression or no depression.
\end{abstract}

Although the frequency and severity of depression and cognitive impairments in Parkinson's disease (PD) have been well characterised by several studies, ${ }^{1-4}$ there is little information on the longitudinal evolution of patients with PD in terms of depression and intellectual decline.

Lieberman et al suggested that in patients with PD depression occurs independently of dementia, and that depression is not a prelude to cognitive impairments, but they provided no empirical data to support this suggestion. Portin and Rinne, in an eight to 10 year longitudinal study, found that $70 \%$ of a series of 79 patients showed a significant deterioration of cognitive function. ${ }^{6}$ Poor response to levodopa, impairments in activities of daily living (ADLs), and a history of thalamotomy were important, albeit moderate, predictors of cognitive decline. ${ }^{7}$ They did not examine the contribution of depression to cognitive decline.

We recently reported the results of the first longitudinal study examining the influence of depression on cognitive decline in patients with PD. ${ }^{8}$ This was a three to four year follow up study of 49 patients with PD (from an original cohort of 70 patients). The main finding was that depressed patients (as defined by a Hamilton depression scale (HDS) score $\geq 7$ ) showed a significantly more severe cognitive decline than non-depressed patients. ${ }^{8}$ Some limitations of that study, however, should be mentioned. A structured psychiatric interview was not carried out, and depressed patients could not be classified into major or minor depression groups. Moreover, neither the decline in ADLs nor the progression of the disease was assessed. Thus for the present study, we examined a larger cohort of patients with PD, and used the present state examination ${ }^{9}$ and DSM-III criteria ${ }^{10}$ to diagnose depression. Patients were divided into those with major, minor, or no depression, and the outcome at one year in terms of cognitive decline, impairment in ADLs, and progression of PD was examined.

\section{Material and methods \\ Study population}

Patients included in this study belong to a cohort of 105 consecutive patients with idiopathic PD who attended the neurology clinic at the Johns Hopkins Hospital, Baltimore. Patients attending the clinic were being seen for neurological evaluation or management of their disorder at regular follow up visits. None of the patients was seen in a specialised clinic for movement disorders. Only one patient refused evaluation. Three patients who could not make their scheduled appointments because of poor health were interviewed in their homes. None of the patients included in this study had a thalamotomy. (A detailed description of this population is provided elsewhere $\left.{ }^{11}\right)$. Follow up evaluations were carried out in $92(88 \%)$ of the 105 patients.

\section{Neurological examination}

The neurological examination was carried out by one of us (TJP) who was blind to the neuropsychiatric data. The age at onset of movement disorder was documented for each patient by interviewing the patient and one relative who lived with the patient. All patients included in this study had hospital records, which were examined to corroborate the information obtained during the interview. The neurological evaluation consisted of a comprehensive neurological examination and a rating scale for symptoms of $\mathrm{PD}$. The rating scale quantified the presence and severity of tremor, rigidity, and akinesia. Scores ranged from 0 to 3 , and left and right limbs were scored independently. Activities of daily living were assessed using the Northwestern Disability Scale (NWDS). ${ }^{12}$ On the basis of clinical findings, the stage of illness was determined using the Hoehn and Yahr stages. ${ }^{13}$

\section{Psychiatric examination}

The psychiatric examination was carried out by one of us (SES) who was blind to the neurological examination and to the data gathered during the initial evaluation. After giving informed consent, patients were administered a series of standardised quantitative 
measures of mood, cognitive function, and social connectedness. Examinations were carried out in a private room between 10 am and $2 \mathrm{pm}$ to minimise any possible effect of diurnal mood variation. Four instruments were used.

The Present State Examination (PSE) (9th edition $)^{9}$ is a semistructured psychiatric interview that elicits symptoms related to depression and anxiety, and is scored by the examiner. Possible scores range from 0 to 144 . On the basis of symptoms elicited with this examination a psychiatric diagnosis was made using DSM-III symptom criteria for major depression or minor depression (that is, dysthymic disorder excluding the two years' duration criteria). The method used to convert PSE symptoms to DSM-III major or minor depression diagnosis was discussed in a previous publication. ${ }^{14}$ Using this instrument, we have recently shown the specificity of affective and autonomic symptoms of depression in PD.

Briefly, the criteria for major depression were dysphoric mood and at least four of the following six symptoms: poor appetite or appreciable weight loss; insomnia; loss of interest or pleasure in usual activities or decrease in sexual drive; feelings of worthlessness, self-reproach, or excessive or inappropriate guilt; complaints or evidence of diminished ability to think or concentrate, such as slowed thinking, or indecisiveness; recurrent thoughts of death, suicidal tendencies, wishes to be dead, or a suicide attempt. The criteria for minor depression (dysthymia) were: dysphoric mood and at least three of the following 10 symptoms: insomnia; feelings of inadequacy, loss of self esteem, or self depreciation; decreased effectiveness or productivity; decreased attention, concentration, or ability to think clearly; social withdrawal; loss of interest in or enjoyment of pleasurable activities; inability to respond with apparent pleasure to praise or rewards; pessimistic attitude toward the future, brooding about past events, or feeling sorry for self; tearfulness or crying; recurrent thoughts of death or suicide.

The Hamilton Rating Scale For Depression ${ }^{15}$ (HAM-D) is a 17 item questionnaire measuring psychological and physiological symptoms of depression, which is filled out by the interviewer. Possible scores range from 0 to 52.
The Mini Mental State Examination (MMSE) $)^{16}$ is an 11 item examination that has been found to be reliable and valid in assessing a limited range of cognitive functions. Scores range from 0 to 30, and scores of 23 or below indicate significant impairment.

The Social Ties Checklist (STC) ${ }^{17}$ quantifies the number of social connections. Scores range from 0 to 10, and higher scores indicate poorer social functioning.

\section{Statistical analysis}

Statistical analysis was carried out with one way analyses of variance (ANOVA). If a significant main effect was found, between group comparisons were carried out with planned $t$ comparisons and non-parametric analysis (Wilcoxon signed rank). Frequency distributions were analysed with $\chi^{2}$ tests with Yates' modification for expected cell sizes below 5 . All $p$ values are two tailed.

\section{Results \\ Demographic findings}

Ninety two of the 105 patients had a follow up examination 12 months after the initial evaluation. Seven of the 13 patients without a one year follow up died during this period, three patients refused a follow up evaluation, and three patients could not be located. No significant differences in terms of neurological or psychiatric characteristics were observed between groups with and without a one year follow up (table 1). Patients without follow up, however, belonged to a lower socio-economic class than patients with a one year follow up.

On the basis of the initial psychiatric diagnosis patients were divided into major, minor, or non-depressed groups. No significant between-group differences were observed in any of the demographic variables (table 2).

\section{Neurological findings}

There was a significant group effect for duration of illness, which was significantly longer for the major depressed group $(F=4 \cdot 21$, $\mathrm{df}=$ $2,89, \mathrm{p}<0.05$ ) (table 3).

A one-way ANOVA for initial minus follow up NWDS scores (activities of daily living)

Table 1 Demographic and clinical findings for patients with and without a follow up evaluation

\begin{tabular}{|c|c|c|c|c|}
\hline & Follow up & No follow up & $t$ or $\chi^{2}$ value & $p$ value \\
\hline $\begin{array}{l}\text { Number of patients } \\
\text { Age (mean (SD) years) } \\
\text { Sex (\% female) } \\
\text { Socioeconomic status (\% Hollingshead class I-III) } \\
\text { Education (mean (SD) years) } \\
\text { Personal history of psychiatric disorders (\% positive) } \\
\text { Duration of illness (mean (SD) years) } \\
\text { Northwestern disability score (mean (SD) years) } \\
\text { Tremor (mean (SD) score) } \\
\text { Rigidity (mean (SD) score) } \\
\text { Akinesia (mean (SD) score) } \\
\text { Hoehn-Yahr stages IV-V (\%) } \\
\text { Present State Exam (mean (SD) score) } \\
\text { Hamilton Depression Scale (mean (SD) score) } \\
\text { Mini Mental State Exam (mean (SD) score) } \\
\text { Major depression (\% positive) } \\
\text { Minor depression (\% positive) } \\
\text { Social ties (mean (SD) scores) }\end{array}$ & $\begin{array}{l}92 \\
96(9 \cdot 3) \\
40 \\
52 \\
13 \cdot 2(3 \cdot 4) \\
10 \\
9 \cdot 7(6 \cdot 4) \\
12 \cdot 0(9 \cdot 1) \\
2 \cdot 5(2 \cdot 2) \\
3 \cdot 4(1 \cdot 8) \\
3 \cdot 4(2 \cdot 0) \\
35 \\
9 \cdot 0(7 \cdot 9) \\
6 \cdot 1(5 \cdot 7) \\
27 \cdot 4(3 \cdot 6) \\
20 \\
21 \\
2 \cdot 9(1 \cdot 5)\end{array}$ & $\begin{array}{l}13 \\
69(7 \cdot 8) \\
54 \\
15 \\
12 \cdot 1(3 \cdot 9) \\
23 \\
8 \cdot 5(6 \cdot 2) \\
11 \cdot 8(11 \cdot 7) \\
2 \cdot 3(2 \cdot 2) \\
3 \cdot 9(1 \cdot 7) \\
4 \cdot 0(2 \cdot 0) \\
38 \\
11 \cdot 5(11 \cdot 2) \\
7 \cdot 2(7 \cdot 5) \\
26 \cdot 5(4 \cdot 9) \\
31 \\
15 \\
2 \cdot 7(2 \cdot 4)\end{array}$ & $\begin{array}{l}1.17 \\
0.86 \\
6.18 \\
1.05 \\
1.98 \\
0.63 \\
0.08 \\
0.30 \\
0.92 \\
0.90 \\
0.06 \\
0.77 \\
0.51 \\
0.61 \\
0.86 \\
0.19 \\
0.33\end{array}$ & $\begin{array}{l}0.25 \\
0.35 \\
0.01 \\
0.30 \\
0.15 \\
0.53 \\
0.93 \\
0.76 \\
0.36 \\
0.38 \\
0.79 \\
0.45 \\
0.61 \\
0.55 \\
0.35 \\
0.65 \\
0.74\end{array}$ \\
\hline
\end{tabular}


Table 2 Demographic findings

\begin{tabular}{|c|c|c|c|c|c|}
\hline & Major depression & Minor depression & No depression & $F$ or $\chi^{2}$ value & $p$ value \\
\hline $\begin{array}{l}\text { Number of patients } \\
\text { Age (mean (SD) years) } \\
\text { Sex (\% female) }\end{array}$ & $\begin{array}{l}18 \\
64 \cdot 5(9 \cdot 3) \\
56\end{array}$ & $\begin{array}{l}19 \\
67 \cdot 6(10 \cdot 8) \\
32\end{array}$ & $\begin{array}{l}55 \\
67 \cdot 2(8 \cdot 9) \\
38\end{array}$ & $\begin{array}{l}0.64 \\
2 \cdot 44\end{array}$ & $\begin{array}{l}0.52 \\
0.29\end{array}$ \\
\hline $\begin{array}{l}\text { Socioeconomic status (\% } \\
\text { Hollingshead class I-III) } \\
\text { Education (mean (SD) years) }\end{array}$ & $\begin{array}{ll}50 \\
12 \cdot 3 \quad(3 \cdot 2)\end{array}$ & $\begin{array}{l}68 \\
13 \cdot 5(4 \cdot 1)\end{array}$ & $\begin{array}{l}49 \\
13.6(3 \cdot 3)\end{array}$ & $\begin{array}{l}2 \cdot 21 \\
0.96\end{array}$ & $\begin{array}{l}0.33 \\
0.38\end{array}$ \\
\hline $\begin{array}{l}\text { Family history of psychiatric } \\
\text { disorders (\% positive) } \\
\text { Personal history of psychiatric }\end{array}$ & 17 & 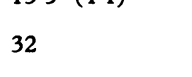 & 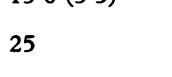 & $1 \cdot 11$ & 0.57 \\
\hline $\begin{array}{l}\text { disorders ( } \% \text { positive) } \\
\text { Handedness ( } \% \text { right handed) }\end{array}$ & $\begin{array}{l}17 \\
89\end{array}$ & $\begin{array}{l}11 \\
95\end{array}$ & $\begin{array}{r}7 \\
93\end{array}$ & $\begin{array}{l}1 \cdot 37 \\
0.47\end{array}$ & $\begin{array}{l}0.50 \\
0.78\end{array}$ \\
\hline
\end{tabular}

showed significant between group differences $(F=3.26, \mathrm{df}=2,89, \mathrm{p}<0.05)$. On individual comparisons, patients with major depression showed a significantly greater decline in ADLs than patients with minor depression (Fisher PLSD $=2.56, \mathrm{p}<0.05$ ) or no depression (Fisher PLSD $=2.13, \mathrm{p}<0.05)$ (table 3). Significant differences between initial and follow up NWDS scores for major depressed patients were also seen after nonparametric statistical analysis (Wilcoxon signed rank $Z=$ $1.96, \mathrm{p}<0.05)$.

Since patients with major depression had a significantly longer duration of illness than patients with minor or no depression, patients with major depression $(n=17)$ were matched with non-depressed patients for duration of illness (within two years) (one patient with major depression could not be matched with a non-depressed patient and was excluded). A paired $t$ test still showed significant declines in ADLs in the group with major depression (paired $t=2.50, \mathrm{df}=16, \mathrm{p}<0.05$ ).

Although between group differences in the initial NWDS scores were not significant, the group with major depression had higher scores than the non-depressed group. To control for this confounding factor, patients with major depression $(n=17)$ were matched with nondepressed patients $(n=17)$ for initial NWDS scores (within three points) (one patient with major depression could not be matched with a non-depressed patient and was excluded). A paired $t$ test still showed a significant decline in ADLs in the group with major depression (paired $t=2.31$, df $=16, \mathrm{p}<0.05$ ).

When changes in NWDS scores of patients with a major depression at the initial evaluation and who still had a major depression at the one year follow up $(n=10)$ were compared with those of patients who had major depression at the initial evaluation but did not have a major depression at one year follow up $(n=8)$ no significant between group differences were found.

A one way ANOVA for initial minus follow up Hoehn and Yahr stage showed significant between group differences $(F=5.46$, df $=$ $2,89, \mathrm{p}<0.01)$. On individual comparisons, patients with major depression showed a faster progression of the illness than patients with no depression (Fisher PLSD $=0.32, \mathrm{p}<0.05$ ) (table 3). By the time of the one year follow up, 10 of the 15 patients $(67 \%)$ with major depression who were not in stage 5 had progressed to the next Hoehn and Yahr stage, compared with 7 of 17 patients (41\%) with minor depression, and 10 of 49 patients $(20 \%)$ with no depression $\left(\chi^{2}=11 \cdot 6, \mathrm{df}=2, \mathrm{p}<0.01\right)$ (table 3). When patients with minor depression and non-depressed patients were pooled the differences with major depressed patients remained significant $\left(\chi^{2}=32 \cdot 1\right.$, df $=1, p<$ $0.0001)$. Finally, when these data were analysed with more appropriate non-parametric statistics (Kruskal-Wallis ANOVA) similar findings emerged $(\mathrm{H}=3.9, \mathrm{p}>0.10$ at initial evaluation, $\mathrm{H}=12.6, \mathrm{p}<0.01$ ) at one year follow up).

During the follow up period seven patients were taking anticholinergics (three in the nondepressed group, two with minor depression, and two with major depression).

No significant between group differences were found in initial minus follow up scores of tremor, rigidity, and akinesia (table 3).

Table 3 Neurological findings

\begin{tabular}{|c|c|c|c|}
\hline & Major depression & Minor depression & No depression \\
\hline$\overline{\text { Duration of illness (mean (SD) years) }}{ }^{\star}$ & $13 \cdot 5(6 \cdot 2)$ & $9 \cdot 4(6 \cdot 2)$ & $8 \cdot 6(6 \cdot 2)$ \\
\hline Levodopa dosage (mean (SD) $\mathrm{mg}$ ): & & & \\
\hline $\begin{array}{l}\text { Initial evaluation } \\
\text { Follow up evaluation }\end{array}$ & $\begin{array}{ll}955 & (392) \\
961 & (352)\end{array}$ & $\begin{array}{ll}839 & (555) \\
832 & (556)\end{array}$ & $\begin{array}{ll}766 & (788) \\
650 & (567)\end{array}$ \\
\hline North western disability (mean (SD) score): & & & \\
\hline $\begin{array}{l}\text { Initial evaluation } \\
\text { Follow up evaluation }\end{array}$ & $\begin{array}{l}16 \cdot 0(11 \cdot 6) \\
18 \cdot 7(12 \cdot 8)\end{array}$ & $\begin{array}{l}13.9(7 \cdot 6) \\
13 \cdot 4(8 \cdot 5)\end{array}$ & $\begin{array}{l}9.9(8 \cdot 1) \\
9.8(8.5)\end{array}$ \\
\hline Tremor (mean (SD) score): & & & \\
\hline $\begin{array}{l}\text { Initial evaluation } \\
\text { Follow up evaluation }\end{array}$ & $\begin{array}{l}2 \cdot 4(2 \cdot 3) \\
2 \cdot 5(2 \cdot 9)\end{array}$ & $\begin{array}{l}3 \cdot 2(2 \cdot 4) \\
2 \cdot 9(2 \cdot 1)\end{array}$ & $\begin{array}{l}2 \cdot 4(2 \cdot 1) \\
2 \cdot 7(2 \cdot 2)\end{array}$ \\
\hline Rigidity (mean (SD) score): & & & \\
\hline $\begin{array}{l}\text { Initial evaluation } \\
\text { Follow up evaluation }\end{array}$ & $\begin{array}{l}3.4(1 \cdot 5) \\
4 \cdot 1(1 \cdot 9)\end{array}$ & $\begin{array}{l}3.6(1.9) \\
3.9(1.7)\end{array}$ & $\begin{array}{l}3.4(1.9) \\
3.7(1.7)\end{array}$ \\
\hline Akinesia (mean (SD) score) & & & \\
\hline $\begin{array}{l}\text { Initial evaluation } \\
\text { Follow up evaluation }\end{array}$ & $\begin{array}{l}3 \cdot 7(2 \cdot 0) \\
4 \cdot 9(2 \cdot 0)\end{array}$ & $\begin{array}{l}3 \cdot 8(1 \cdot 9) \\
4 \cdot 7(1 \cdot 6)\end{array}$ & $\begin{array}{l}3.3(2 \cdot 1) \\
3.9(1 \cdot 8)\end{array}$ \\
\hline Hoehn and Yahr (mean (SD) stage) & & & \\
\hline $\begin{array}{l}\text { Initial evaluation } \\
\text { Follow up evaluation } \star \star\end{array}$ & $\begin{array}{l}3.2(1.4) \\
3.9(1.2)\end{array}$ & $\begin{array}{l}3 \cdot 1(1 \cdot 2) \\
3 \cdot 6(1 \cdot 0)\end{array}$ & $\begin{array}{l}2 \cdot 6(1 \cdot 2) \\
2 \cdot 8(1 \cdot 2)\end{array}$ \\
\hline
\end{tabular}

${ }^{\star}$ Major $v$ minor depression $\mathrm{p}<0.05$; major $v$ no depression $\mathrm{p}<0.05$

$\star \star$ Major $v$ no depression $\mathrm{p}<0.05$. 
Table 4 Psychiatric outcome

\begin{tabular}{llll}
\hline \multirow{4}{*}{ Initial diagnosis } & Diagnosis at 1 year followup & \\
\cline { 2 - 4 } & Major depression & Minor depression & No depression \\
\hline Major depression (\%) & $56(10)$ & $33(6)$ & $11(2)$ \\
Minor depression (\%) & $11(2)$ & $26(5)$ & $63(12)$ \\
No depression (\%) & 0 & $18(10)$ & $82(45)$ \\
\hline Numbers in parenthesis are number of patients.
\end{tabular}

Numbers in parenthesis are number of patients.

Psychiatric findings

Table 4 shows psychiatric diagnosis at one year follow up.

A one way ANOVA for initial minus follow up MMSE scores showed significant between group differences $(F=4 \cdot 36, \mathrm{df}=1,89, \mathrm{p}<$ $0.05)$. On individual comparisons patients with major depression showed significantly greater declines in MMSE scores than patients with minor depression (Fisher PLSD $=1 \cdot 84, p$ $<0.05$ ) and non-depressed patients (Fisher PLSD $=1.52, \mathrm{p}<0.05$ ) (table 4). Fifteen of the 18 patients ( $83 \%$ ) with major depression had lower follow up than initial scores, compared with 29 of the 55 (53\%) non-depressed patients $\left(\chi^{2}=3.90, \mathrm{df}=1, \mathrm{p}<0.05\right)$ and 12 of the 19 patients $(63 \%)$ with minor depression $\left(\chi^{2}=1.90, \mathrm{df}=1, \mathrm{p}>0 \cdot 10\right)$. Significant differences between initial and follow up MMSE scores for patients with major depression were also observed after non-parametric statistical analysis (Wilcoxon signed $\operatorname{rank} Z=$ 14.23, $\mathrm{p}<0.0001$ ).

No significant differences in changes in scores on the MMSE were observed when the group with major depression was divided into those who still had a major depression at one year follow up $(n=10)$ and those with a minor or no depression $(n=8)$.

Finally, no significant between-group differences were observed in initial minus follow up STC scores.

During the follow up period four of the 20 patients with minor depression were taking antidepressant drugs (amityptiline, mean dosage, $21 \mathrm{mg}$ /day). At one year follow up, three of the four patients were non-depressed and the remaining one had a major depression. Four of the 18 patients with major depression were taking antidepressant drugs (amitryptiline; mean dosage $50 \mathrm{mg} /$ day). At one year followup two were non-depressed and the two others still had a major depression. The mean change in NWDS and MMSE scores for the patients with major depression who were taking tricyclic drugs was similar to the rest of the group (initial $v$ follow up) scores on NWDS: $24.2 v$ 28.5; scores on MMSE: $23.0 v 21 \cdot 0$ ).

\section{Discussion}

This study has two main findings. Firstly, in a consecutive series of patients with $\mathrm{PD}$, a 12 month period was sufficient to show a significant progression along the stages of illness, and a significant decline in both cognitive functions and activities of daily living. Secondly, this decline was either specific or significantly greater in patients with a major depression at the initial evaluation than in patients with either minor or no depression.

One limitation of this study is that 13 patients of the original cohort of 105 could not be followed up. However, no significant differences in terms of demographic, neurological, and psychiatric characteristics were observed between patients with and without a one year follow up, although patients without a follow up belonged to a lower socioeconomic class. Another limitation is that our diagnosis of depression may have been influenced by the presence and severity of bradykinesia. In a recent study, however, we found that PD patients with a depressive mood showed a significantly higher frequency of both autonomic and affective symptoms of depression than did PD patients without a depressive mood who were matched for age, education, and duration and stage of illness. ${ }^{18}$ The fact that some depressed patients improved from their depression spontaneously (even though they were more impaired by the disease) suggests that depression in PD cannot be construed as a mere reaction to the deficits but may resemble the cyclical evolution of a "functional" depression.

In a comprehensive study of psychiatric morbidity in patients with PD, Brown and MacCarthy found a widespread psychiatric morbidity, most commonly manifest as simple depression, tension, irritability, worrying, and loss of interest and concentration. ${ }^{19}$ We recently replicated this profile of depressive symptoms in patients with PD. ${ }^{11}$ The present study showed that although patients with major depression had a significantly longer duration of illness and more severe disabilities at the initial evaluation than non-depressed patients, they showed a significantly faster cognitive and functional decline even after they were matched with non-depressed patients for duration of illness and initial disability.

Table 5 Psychiatric findings

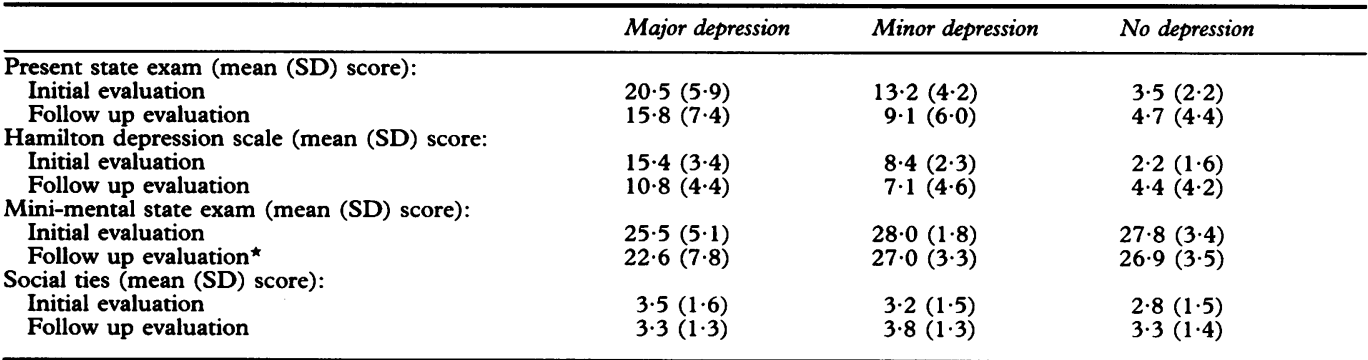

ॠMajor $v$ minor depression $\mathrm{p}<0.05$; major $v$ no depression $\mathrm{p}<0.05$ 
In the first study to examine the influence of depression on the longitudinal evolution of PD, Brown et al mailed self report questionnaires (the Beck depression inventory and an ADLs scale) to 152 patients with PD one year after they had returned similar questionnaires. $^{20}$ About two thirds of the initial cohort returned the follow up questionnaires. Brown et al found the greatest decline in ADL scores in patients who became depressed during the follow up period. Cognitive decline was not examined. In both Brown et al's study and our previous study, ${ }^{8}$ patients were divided into depressed and non-depressed groups based on a cut off score on the depression scale, and standardised criteria for depression, such as the DSM-III, were not used. The present study provides some validation for distinguishing major from minor depression, since these disorders have a significantly different longitudinal evolution.

The question that now arises is, what is the mechanism (or mechanisms) underlying the different longitudinal evolution of PD on the basis of the presence or absence of major depression? Our previous longitudinal study suggested a continuum from no, to mild, to severe depression and concomitant degrees of cognitive impairment. ${ }^{8}$ Recent evidence which supports this suggestion has been provided by Torack and Morris, who found that patients with PD, cognitive impairment, and depression had widespread pathological changes in the ventral tegmental area which contains the cell bodies of dopaminergic fibres projecting to limbic related cortical and subcortical regions. ${ }^{21}$ On the basis of laboratory and clinical data, Cools suggested that patients with PD and no depression or dementia had a normally functioning dopaminergic mesocortico-limbic system, while the opposite was true for patients with $\mathrm{PD}$, depression, and cognitive impairments. $^{22}$ Finally, Cantello et al have recently shown a lack of euphoric response to methylphenidate in patients with $\mathrm{PD}$ and depression but not in patients with PD without depression, suggesting a significant derangement of reward related dopaminergic mesocortico-limbic pathways in patients with PD and major depression. ${ }^{23}$

We recently showed the presence of a regional difference in cerebral metabolic activity between PD patients with and without major depression. ${ }^{24}$ Cerebral glucose metabolism using 2-( $\left.{ }^{18} \mathrm{~F}\right)$-fluoro-2-deoxy-D-glucose and positron emission tomography was measured in five patients with PD and depression (four major and one minor) and four patients with $P D$ and no depression. Both groups were similar in age, duration of $\mathrm{PD}$, and stage of illness. There was a significantly lower metabolic activity in the head of the caudate and the inferior frontal cortex in the depressed than in the non-depressed patients. There also was a significant inverse correlation between relative glucose metabolism in the inferior frontal cortex and depression scores (that is, the lower the metabolic activity in inferior frontal regions, the higher the depression score). It should be noted, however, that in the present study some patients with PD and depression improved either spontaneously or on antidepressant treatment. Thus whether our metabolic findings in depressed patients are "state" or "trait" dependent still remains to be examined.

Further evidence for a biological difference between depressed and non-depressed patients with PD stems from the studies of Mayeux $e t$ al..$^{25-27}$ They reported a significantly lower concentration of 5-hydroxyindoleacetic acid (5-HIAA) in the cerebrospinal fluid of depressed than in non-depressed patients with PD. ${ }^{28}$ They also found that patients who were both depressed and demented had the lowest 5-HIAA concentrations, and suggested that the association of dementia and depression in PD may represent a unique clinical entity. ${ }^{27}$ Their speculation that the occurrence of depression in PD may increase the probability of cognitive impairments later in the course of the disease ${ }^{27}$ is supported by our present findings. Psychological factors may also underlie the faster progression of cognitive and physical disabilities in PD patients with major depression. As Brown and MacCarthy suggested, "The link between psychiatric morbidity and disability may reflect the fact that the individual's psychiatric status itself contributes to the overall level of disability."19 Future studies should further examine this interesting hypothesis.

In conclusion, we replicated in a larger population our previous finding of a faster cognitive decline among depressed than nondepressed patients with PD. The present study showed that this decline was significantly greater in patients with a major depression. It also showed that major depressed patients with PD had a significantly greater decline in ADLs and that they progress significantly faster through the stages of the disease than minor depressed or non-depressed patients. Since quantitative but not qualitative differences in the pattern of change during the one year follow up period have been shown between patients with major depression and those with either minor or no depression, our data suggests a continuum from no, to mild, to severe depression and concomitant degrees of severity of PD and cognitive impairment. It is hoped that future studies may illuminate the neuropathological and neurochemical changes underlying the association of depression, cognitive impairments, and $P D$, and determine the reversibility of these impairments after antidepressant treatment.

This work was supported in part by the following National Institutes of Health grants: Research Scientist Award MH00163 (RGR), NS-15178, NS-15080, and MH-40355. This work was also supported in part by a grant from the Institute of Neurological Investigation Raul Carrea (SES), and a grant from the National Alliance for Research in Schizophrenia and Depression (SES).

1 Sano M, Stern Y, Cote L, Williams JB, Mayeux R Depression in Parkinson's disease: a biochemical model. $\mathcal{f}$ Neuropsychiatry 1990;2:88-92.

2 Taylor AE, Saint-Cyr JA. Depression in Parkinson's disease: reconciling physiological and psychological perspectives. 7 Neuropsychiatry 1990;2:92-8. 
3 Stern Y, Mayeux R. Intellectual impairment in Parkinson's disease. Adv Neurol 1986;45:405-8.

4 Brown RG, Marsden CD. Cognitive function in Parkinson's

Cription to theory. Dziatolowski M, Kupersmith M, Serby M, Goodgold A, Korein J, Goldstein M. Dementia in Parkinson's disease. Ann Neurol 1979;6:355-9.

6 Portin R, Rinne UK. Neuropsychological responses of parkinsonian patients to long-term levedop treatment. parkinsonian patients to long-term levedopa treatment. disease: current progress, problems and management. Amsterdisease: current progress, problems

7 Portin R, Rinne UK. Predictive factors for cognitive deterioration and dementia in PD. Adv Neurol 1986; 45:413-6.

8 Starkstein SE, Bolduc PL, Mayberg HS, Preziosi TJ, Robinson RG. Cognitive impairments and depression in Parkinson's disease: a follow-up study. $\mathcal{F}$ Neurol Neurosurg Psychiatry 1990;53:597-602.

9 Wing JK, Cooper JE, Sartorius N. Measurements and classification of psychiatric symptoms. Cambridge: Cambridge University Press, 1974

10 American Psychiatric Association. Diagnostic and Statistical Manual of Mental Disorders (DSM-III). 3rd ed. WashManual of Mental Disorders (DSM-III). 3rd

11 Starkstein SE, Preziosi TJ, Bolduc PL, Robinson RG. Depression in Parkinson's disease. $\vec{f}$ Nerv Ment Dis $1990 ; 178: 27-31$.

12 Canter GJ, de la Torre R, Mier M. A method for evaluating disability in patients with Parkinson's disease. $\mathcal{F}$ Nerv Ment Dis 1961;133:143-7.

13 Hoehn MM, Yahr MD. Parkinsonism: onset, progression and mortality. Neurology 1967;17:427-42.

14 Robinson RG, Kubos KI, Starr LB, Rao K, Price TR. Mood changes in stroke patients: relationship to lesion location. Compr Psychiatry 1983;24:555-66.

15 Hamilton MA. A rating scale for depression. $f$ Neurl Neurosurg Psychiatry 1960;23:56-62.

16 Folstein MF, Folstein SE, McHugh PR. "Mini-mental state": a practical method for grading the cognitive state for the clinician. $f$ Psychiatr Res 1975;12:189-98.

17 Starr LB, Robinson RG, Price TR. Reliability, validity, and clinical utility of the social functioning exam in the assessment of stroke patients. Exp Aging Res 1983;9: $101-6$.

18 Starkstein SE, Preziosi TJ, Forrester AW, Robinson RG. Specificity of affective and autonomic symptoms of depression in Parkinson's disease. 7 Neurol Neurosurg Psychiatry 1990;53:869-73.

19 Brown RG MacCarthy B. Psychiatric morbidity in patients with Parkinson's disease. Psychol Med 1990;20:77-87.

20 Brown RG, MacCarthy B, Gotham AM, Der GB, Marsden CD. Depression and disability in Parkinson's disease: a CD. Depression and disability in Parkinson's disease:

21 Torack RM, Morris JC. The association of ventral tegmental area histopathology with adult dementia. Arch Neurol 1988;45:497-501.

22 Cools AR. Role of neostriatal and mesostriatal or mesolimbic dopaminergic fibers in Parkinson's disease with and without dementia: prospects, concepts and facts. fap I Psychopharmacology 1991 (in press).

23 Cantello R, Aguggia M, Gilli M, Belsedime M, Cutin IC, Riccio $A$ et al. Major depression in Parkinson's disease possible role of the hedonic dopamine synapse. 7 Neurol Neurosurg Psychiatry 1989;52:724-31.

24 Mayberg HS, Starkstein SE, Sadzot B, Preziosi T, Andrezejewski PL, Dannals RF, et al. Selective inferior frontal lobe hypmetabolism in depressed patients with Parkinson's disease. Ann Neurol 1990;28:57-64.

25 Mayeux $R$ Stern Y, Williams JBW, Cote L, Franz A Dyrenfurth I. Clinical and biochemical features of depression in Parkinson's disease. Am $\mathcal{F}$ Psychiatry 1986 143:756-9.

26 Mayeux R, Stern M, Williams JBW, Cote LJ. The relationship of serotonin to depression in Parkinson's disease. Movement Disonders 1988;3:237-44.

27 Sano M, Stern Y, Williams J, Cote L, Rosenstein R, Mayeux $R$ Coexisting dementia and depression in Parkinson's disease. Arch Neurol 1989;46:1284-6. 